\title{
Plantas útiles en Huertos Familiares Tradicionales de Xalpatláhuac, Región Montaña de Guerrero
}

\author{
Useful plants in Traditional Family Gardens of Xalpatláhuac, \\ Mountain Region of Guerrero
}

\author{
Viviana Paulina Jiménez-Alpizar ${ }^{*}$, Manuel Melesio-Velázquez ${ }^{1,2}$, Itandehui Martínez-Rodríguez ${ }^{1}$ \\ ${ }^{1}$ Departamento de Biología, Facultad de Ciencias, Universidad Nacional Autónoma de México, México, \\ ${ }^{2}$ Departamento de Etnología, Escuela Nacional de Antropología e Historia, México \\ * Autora a quien se dirige la correspondencia: vivianajim@ciencias.unam.mx
}

Recibido: 26 de noviembre de 2020 / Aceptado: 5 de mayo de 2021

\section{Resumen}

T os huertos familiares tradicionales son uno de los sistemas agroforestales más frecuentes en el México, ya que a ¿través de ellos la población puede obtener distintos beneficios, principalmente servicios ecosistémicos, así como recursos para su alimentación, venta e intercambio, ornamentación, así como la preservación de prácticas tradicionales de manejo de los recursos naturales. En la Región Montaña de Guerrero, los huertos familiares cumplen diferentes funciones importantes para la obtención de los recursos en mención. Los objetivos de esta investigación fueron identificar la diversidad de plantas útiles en los huertos familiares tradicionales, determinar la frecuencia de utilización de dichas plantas y establecer sus categorías de uso. Esto a partir de encuestas y entrevistas semiestructuradas, visitas a los huertos de traspatio y observación directa, con las cuales se logró identificar 104 especies ubicadas en cinco categorías: alimenticias, medicinales, ornamentales, mágico-religiosas, y combustibles. Siendo las de carácter alimenticio y medicinal las más abundantes y representativas, siendo un complemento importante para el consumo familiar dentro de la comunidad. Con este trabajo se reafirmó el nivel organizativo de las familias y la necesidad de complementar sus actividades cotidianas con productos obtenidos del huerto familiar y no únicamente con productos obtenidos del campo.

Palabras clave: Sistemas agroforestales, diversidad biológica, manejo de los recursos naturales, aprovechamiento de los recursos naturales, etnoecología

\section{Abstract}

$\mathrm{T}$ raditional home gardens are one of the most frequent agroforestry systems in Mexico, since through them the population can obtain different benefits, mainly ecosystem services, as well as resources for their food, sale and exchange, ornamentation, as well as preservation. of traditional natural resource management practices. In the Mountain Region of Guerrero, home gardens fulfill different important functions to obtain the resources in question. The objectives of this research were to identify the diversity of useful plants in traditional home gardens, determine the frequency of use of these plants and establish their categories of use. This from surveys and semi-structured interviews, visits to backyard gardens and direct observation, with which it was possible to identify 104 species located in five categories: food, medicinal, ornamental, magical-religious, and fuel. Being those of a nutritional and medicinal nature the most abundant and representative, being an important complement for family consumption within the community. This work reaffirmed the organizational level of the families and the need to complement their daily activities with products obtained from the family garden and not only with products obtained from the field.

Keywords: Agroforestry systems, biological diversity, natural resource management, use of natural resources, ethnoecology 


\section{Introducción}

México es considerado como uno de los países con mayor patrimonio biocultural, debido a que alberga al $10 \%$ de la riqueza biológica del planeta y el quinto lugar en riqueza de lenguas indígenas a nivel mundial; el patrimonio biocultural surge de la necesidad de reconocer y valorar la relación hombre-naturaleza, abarcando desde el conocimiento y el uso tradicional de la biodiversidad hasta los valores espirituales, aspectos que son transmitidos de generación en generación (Instituto de Ecología [INECOL], 2021). Del mismo modo, se reconoce la presencia de sistemas de agricultura tradicional en el país, algunos incluso de origen prehispánico, como es el caso de la agricultura de campos elevados o drenados, las terrazas o los huertos familiares de los cuales pueden encontrarse referentes en distintas partes del país (Moreno Calles et al., 2016). La expresión de estas diversidades (biológica y cultural) se encuentra reflejada en los sistemas agroforestales tradicionales (SAFT) a través de la domesticación, el manejo y el aprovechamiento de los recursos forestales maderables y no maderables, cuyo manejo in situ ha inducido ajustes ambientales que satisfacen necesidades sociales y económicas (Chávez et al., 2002). En estos sistemas interactúa la biodiversidad silvestre, la biodiversidad domesticada y los seres humanos dentro de un contexto cultural (Figura 1).

\section{Figura 1}

Diagrama de un sistema agroforestal

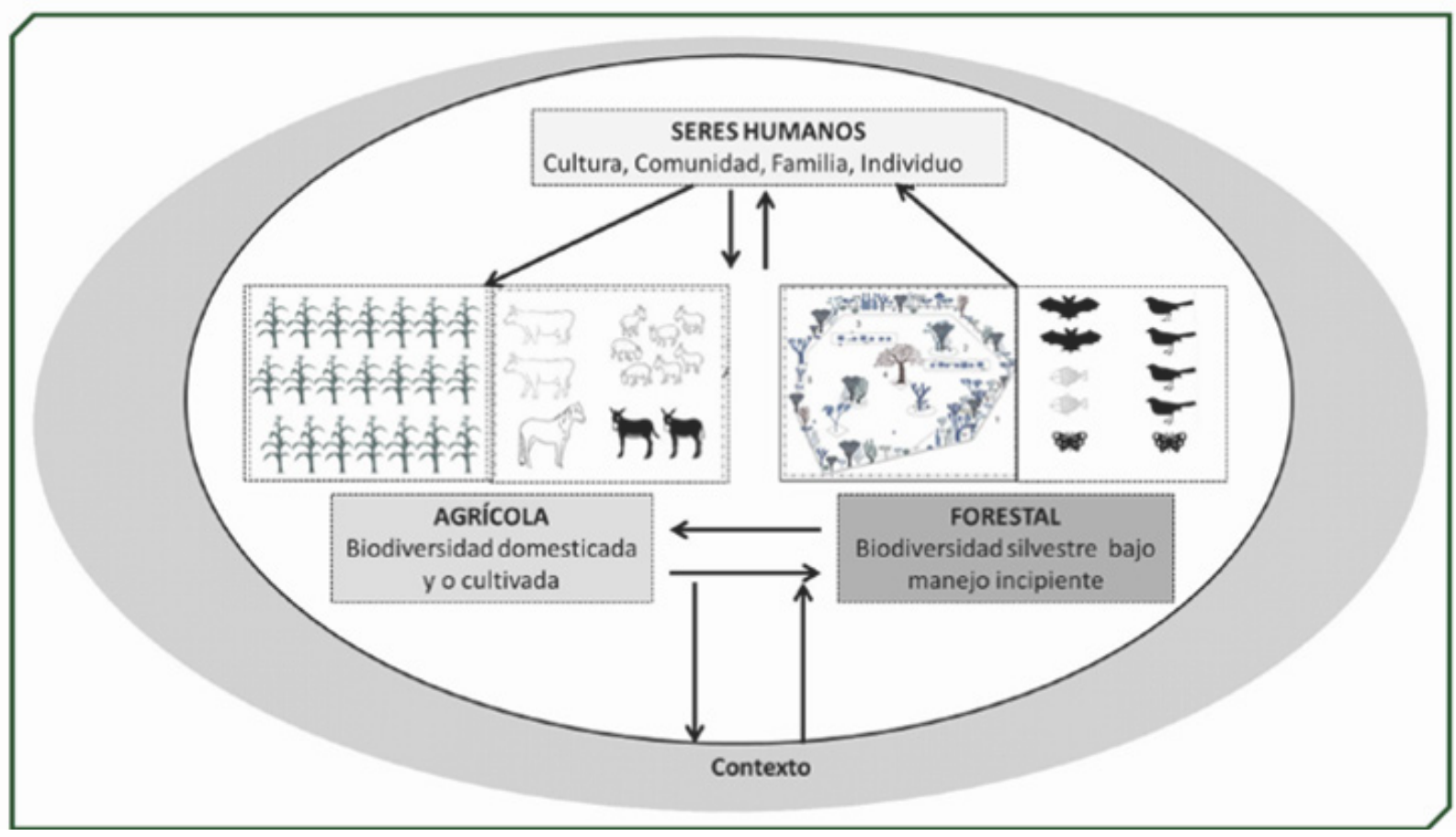

Nota. Se ejemplifican los componentes agrícolas y forestales del sistema y la dirección humana. Adaptado de "La etnoagroforestería: El estudio de los sistemas agroforestales tradicionales de México” por A. I. Moreno-Calles, V. J. Galicia-Luna, A. Casas, V. M. Toledo, M., Vallejo-Ramos, D. Santos-Fita \& A. Camou-Guerrero, 2014, p. 3. 
En la composición de los SAFT se encuentran especies nativas silvestres, con las que se contrarresta la erosión genética a través del desarrollo de procesos continuos de domesticación, convirtiéndolos en espacios con una gran capacidad de mantener la biodiversidad de la región donde se encuentre ubicado el sistema, además de ser reservorios de elementos culturales a través de las técnicas de manejo tradicional y la relación del ser humano con su entorno (Vallejo-Ramos et al., 2016). Dentro de estos procesos de domesticación se encuentran distintos procesos tradicionales de selección artificial, donde los seres humanos, con sus cuidados, procuran la supervivencia y la reproducción de los organismos de su mayor interés, de acuerdo con los atributos que desde el punto de vista social, económico, cultural, ecológico y tecnológico resulten de su mayor conveniencia (Casas \& Parra, 2016).

En cuanto a los tipos de manejo de los SAFT, el huerto familiar es uno de los más representativos (Moreno-Calles et al., 2016). Este es definido como el reservorio genético vegetal aledaño a la casa-habitación, cuyo establecimiento refleja un aspecto fundamental de la identidad cultural de un grupo humano en relación con la naturaleza; en él se practican actividades culturales, sociales, biológicas y agronómicas, constituyendo una unidad económica de autoconsumo a la puerta del hogar (Gispert et al., 1993). Lo podemos encontrar con distintos nombres como huerto familiar, solar o jardín de traspatio y como bien se menciona, se encuentra situado próximo a la vivienda. Es modificado constantemente para satisfacer las necesidades locales o familiares a corto y mediano plazo, además permite la conservación de una parte de la biodiversidad nativa silvestre. Los huertos familiares tradicionales se encuentran en mayor presencia en zonas rurales o en transición, teniendo una producción no especializada, utilizando los recursos disponibles para complementar su alimentación e ingreso (López Pérez, 2017). Respecto a su conformación (Cano Contreras, 2015) menciona que a un huerto familiar los pueden constituir diversas zonas, entre las cuales se encuentran la casa habitación, áreas de trabajo, áreas para cultivo, áreas de esparcimiento, áreas de intercambio social, entre otro tipo de estructuras propias de los hogares rurales como corrales o bodegas. De esta manera un huerto familiar no necesariamente comprende un único espacio dentro de los hogares, sino que se adapta a los distintos espacios disponibles en los hogares donde se encuentran presentes.
A través de un proyecto gestionado por estudiantes de universidades públicas, compuesto principalmente por estudiantes de la Universidad Nacional Autónoma de México (UNAM), por medio de la Brigada Multidisciplinaria de Apoyo a las Comunidades de México (BMACM) se han generado vínculos con cinco comunidades de la región Montaña de Guerrero, a través del trabajo conjunto en los ejes de salud, educación e identidad cultural. En Xalpatláhuac existen dos tipos de huertos, los huertos familiares tradicionales y los huertos comunitarios de plantas medicinales. Los huertos familiares tradicionales son aquellos que las familias tienen en el hogar, y que pueden estar constituidos por diferentes elementos arquitectónicos (Mariaca Méndez et al., 2010). Por su parte, los huertos comunitarios fueron colocados por el proyecto de Agroecología de la BMACM en lugares clave de la comunidad, su composición vegetal son plantas medicinales populares que no se encuentran con facilidad en sus huertos familiares, en el monte o en el mercado municipal. Los huertos familiares tradicionales se encuentran presentes y de forma muy variable en toda la comunidad; por tanto, el objetivo de este trabajo es conocer la composición vegetal y caracterizar la estructura de los huertos familiares tradicionales a través del estudio de la diversidad de plantas, sus categorías de uso, los factores socioculturales y la infraestructura del huerto familiar tradicional en la comunidad de Xalpatláhuac, Guerrero.

\section{Materiales y métodos}

\section{Ubicación geográfica de la investigación}

La comunidad de Xalpatláhuac xali "arena" y paatlahuac "arenal ancho o amplio" se encuentra ubicada dentro del municipio de Xalpatláhuac, el cual también es cabecera municipal (Instituto Nacional para el Federalismo y el Desarrollo Municipal [INAFED], 2020). Se ubica a una altura promedio de $1,600 \mathrm{msnm}$, el clima es subhúmedo-semicálido, la vegetación predominante es selva baja caducifolia (Rzedowski, 1978) con temperaturas de entre $8^{\circ} \mathrm{C}$ y $29^{\circ} \mathrm{C}$ a lo largo de todo el año.

La región de La Montaña del Estado de Guerrero se caracteriza por ser una de las zonas con mayor concentración de población indígena, la población municipal cuenta con un registro de 11,726 habitantes de 
los cuales 4,255 pertenecen a la cabecera municipal y la comunidad con el mismo nombre perteneciente a la cultura nahua, el $98.13 \%$ de este municipio se considera indígena y un $0.76 \%$ es considerado afrodescendiente (Instituto Nacional de Estadística y Geografía [INEGI], 2015). La tasa de alfabetización según el último registro del INEGI en el año 2015 se puede dividir en dos grandes grupos de 15 a 24 años el 95.2\% alfabetizados y de rango de edad de 25 años y más solo el $48 \%$ son alfabetizados, el $62 \%$ de la población adulta no sabe leer ni escribir lo que genera una barrera de entendimiento social con otras comunidades.

\section{Figura 2}

Localización geográfica de la Comunidad Xalpatláhuac, perteneciente al Municipio Xalpatláhuac dentro de la Región Montaña del Estado de Guerrero, México

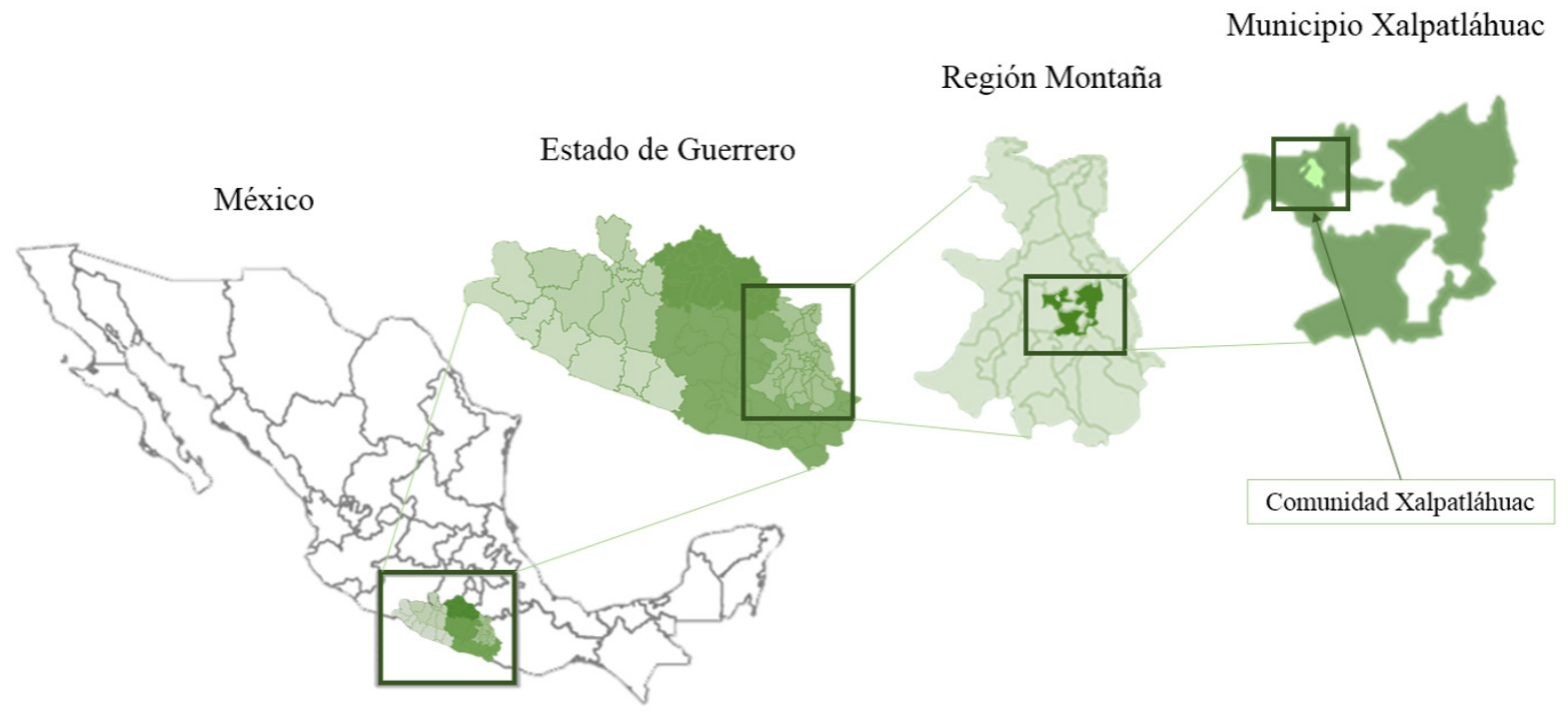

Recolección de datos

El periodo de este trabajo se ubica entre diciembre de 2016 y julio de 2019 , donde se realizaron 6 visitas a la comunidad, cada una con duración de dos semanas en los meses de julio y diciembre de cada año (temporada vacacional escolar). El trabajo se realizó con una muestra representativa de la población, por medio de 81 encuestas, de las cuales 69 son cuestionarios (una encuesta por unidad familiar) y 12 entrevistas semiestructuradas (una encuesta por especialista médico tradicional), ambos formatos incluyen el mismo bloque de preguntas sobre la composición de los huertos familiares tradicionales de Xalpatláhuac.
Técnicas e instrumentos

El muestreo al realizar las encuestas se hizo al azar y cuando se realizaron las entrevistas semiestructuradas se utilizó la técnica de bola de nieve (Ramírez Bacca, 2010). Se realizaron entrevistas semiestructuradas siguiendo la propuesta de Cadena y colaboradores (2017); dirigidas de manera individual o en grupos (Hamui-Sutton \& Varela-Ruiz, 2013) según las situaciones y disposición de los colaboradores. La información recabada se registró de manera escrita y con recursos fotográficos, cuando fue posible. 


\section{Procesamiento y análisis de información}

El bloque de preguntas de huertos familiares de las entrevistas y las encuestas fueron capturadas dentro de una base de datos general en la hoja de cálculo Microsoft Excel, de la cual se obtuvieron matrices básicas de datos (BDM), con las que se realizaron los siguientes análisis cuantitativos:

(a) Para conocer la diversidad de plantas útiles en el huerto familiar se realizó una evaluación de importancia cultural, el análisis de frecuencia de mención por familias botánicas y por especie.

(b) Para conocer la distribución de las plantas útiles por categoría de uso, se realizó el análisis de frecuencia de uso.

La identificación taxonómica se realizó con el uso de claves dicotómicas, la verificación de los nombres científicos fue a través de la plataforma de PlantList y correlacionando con catálogos de plantas útiles de la Región Montaña y de la Biblioteca Digital de la Medicina Tradicional Mexicana.

Con el fin de facilitar la interpretación de los resultados, se establecieron cuatro categorías de análisis, divididas a su vez en subcategorías: uso (comestible, medicinal, ornamental, mágico-religiosas y maderable), infraestructura (composición vegetal y elementos estructurales del huerto), economía (comercio e intercambio) y prácticas socioculturales (identidad, género $\mathrm{y}$ transmisión del conocimiento).
Las categorías de uso se construyeron con los datos de aprovechamiento a cada planta, es decir, si la planta tenía tres usos distintos (por ejemplo: comestible, medicinal y ornamental) se le considera en las tres categorías. Para conocer las categorías de uso representativas o más abundantes, se hace una sumatoria de las especies que se encuentran en cada una y se calcula en porcentaje.

Nos apegamos en todo momento de acuerdo al Código de Ética para la Investigación-Acción y la Colaboración Etnocientífica en América Latina (Sociedad Latinoamericana de Etnobiológica, 2016). Cumplimos con las normas de la comunidad, pidiendo permiso a las autoridades locales con un acuerdo de consentimiento informado y uso de información (Anexo 1), las cuales fueron autoridades religiosas, el tlayakanki (autoridad tradicional) y la presidencia municipal.

\section{Resultados}

Diversidad de plantas útiles

Se obtuvo un total de 125 nombres comunes de plantas útiles. Sin embargo, para 21 de ellas no se encontró correspondencia (asociación) con alguna especie identificada, para el resto se encontró una relación con 104 especies, distribuidas en 42 familias botánicas (Figura 3), donde las familias Asteraceae (15 spp), Lamiaceae (9 spp), Rutaceae (9 spp), Solanaceae (7 spp) y Lauraceae (6 spp) son las que presentan un mayor número de especies.

\section{Figura 3}

Frecuencia de plantas por familia botánica en los huertos familiares tradicionales de Xalpatláhuac, Guerrero

Familias botánicas presentes en los Huertos Familiares

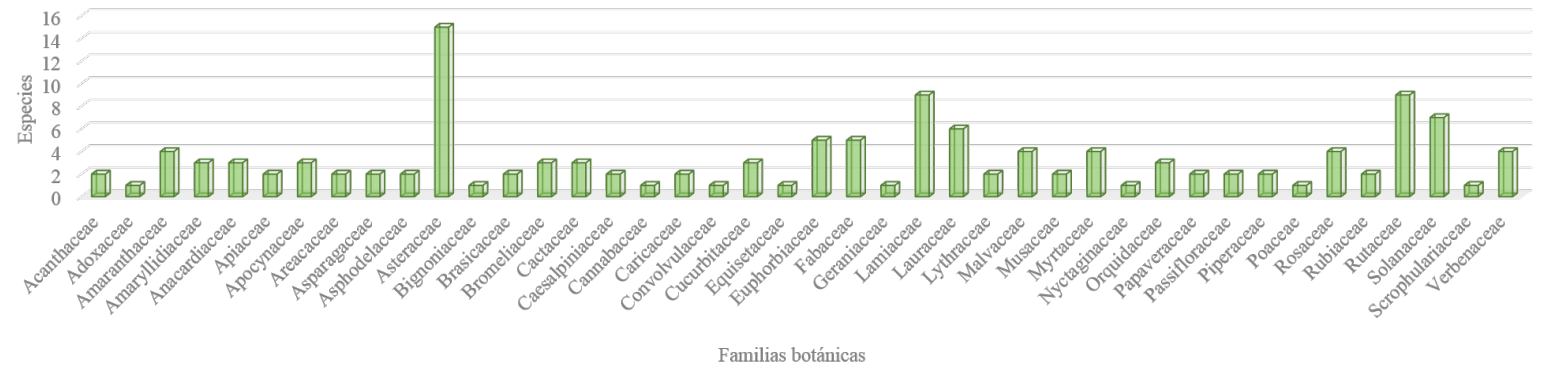


Las especies con mayor presencia en los huertos familiares tradicionales por familia botánica, mencionadas según su porcentaje de mención son: Asteraceae con Calea ternifolia (Zacatechichi), Tagetes erecta (Cempasúchil), Artemisa ludoviciana (Estafiate), Senecio $s p$. (Azumiate), Arnica sp. (Árnica) y Matricaria recutita (Manzanilla); Lamiaceae con Ocimum $s p$. (Albahaca), Menta piperita (Hierbabuena), Marrubium vulgare (Manrubio), Rosmarinus officinalis (Romero) y Melissa officinalis (Toronjil); Rutaceae con Ruta chalepensis (Ruda), Citrus limon (Limón), Casimiroa sp. (Zapote dormilón) y Citrus sinensis (Naranja); Solanaceae con Solanum lycopersicum (Tomate), Physalis philadelphica (Jitomate) y Capsicum annuиm (Chile guajillo); Lauraceae con Cinnamomun $s p$. (Canela), Cinnamomun camphora (Alcanfor) y Persea sp. (Aguacate).

Las especies con una mayor frecuencia de mención por entrevista pertenecen a las categorías de uso "comestible" y "medicinal", como son Anoda cristata (Alache, 86\%), Menta piperita (Hierbabuena, 63\%), Cucurbita pepo (Calabaza, 63\%), Psidium guajava (Guayaba, 60\%), Zea mays (Maíz, 60\%), Marrubium vulgare (Manrubio, 60\%), Phaseolus vulgaris (Frijol,
56\%), Chenopodium ambrosioides (Epazote, 53\%), Matricaria recutita (Manzanilla, 53\%), Amaranthus sp (Quintonil, 53\%), Arnica sp (Árnica, 50\%), Capsicum annuum (Chile de árbol, 46\%), Amphipterygium adstringens (Cuachalalate, 46\%), Calea ternifolia (Zacatechichi, 45\%).

Las especies registradas se ubican en cinco categorías de uso: comestibles, medicinales, ornamentales, mágico-religiosas y maderables. Siendo las de carácter medicinal (54\%) y comestibles (28\%) las más abundantes y diversas (Figura 4).

\section{Discusión}

Diversidad de plantas útiles

La salud y la nutrición en todo momento van de la mano y dentro de la comunidad de Xalpatláhuac esto puede observarse en la proporción representada bajo especies con usos medicinales, las cuales ocupan el $72 \%$ de plantas útiles que conforman los huertos familiares tradicionales de la comunidad, colocándola como la categoría de uso más representativa y diversa (Tabla 1).

\section{Figura 4}

Porcentaje representado por cada categoría de uso de las plantas presentes en los huertos familiares tradicionales de Xalpatlahuac, Guerrero.

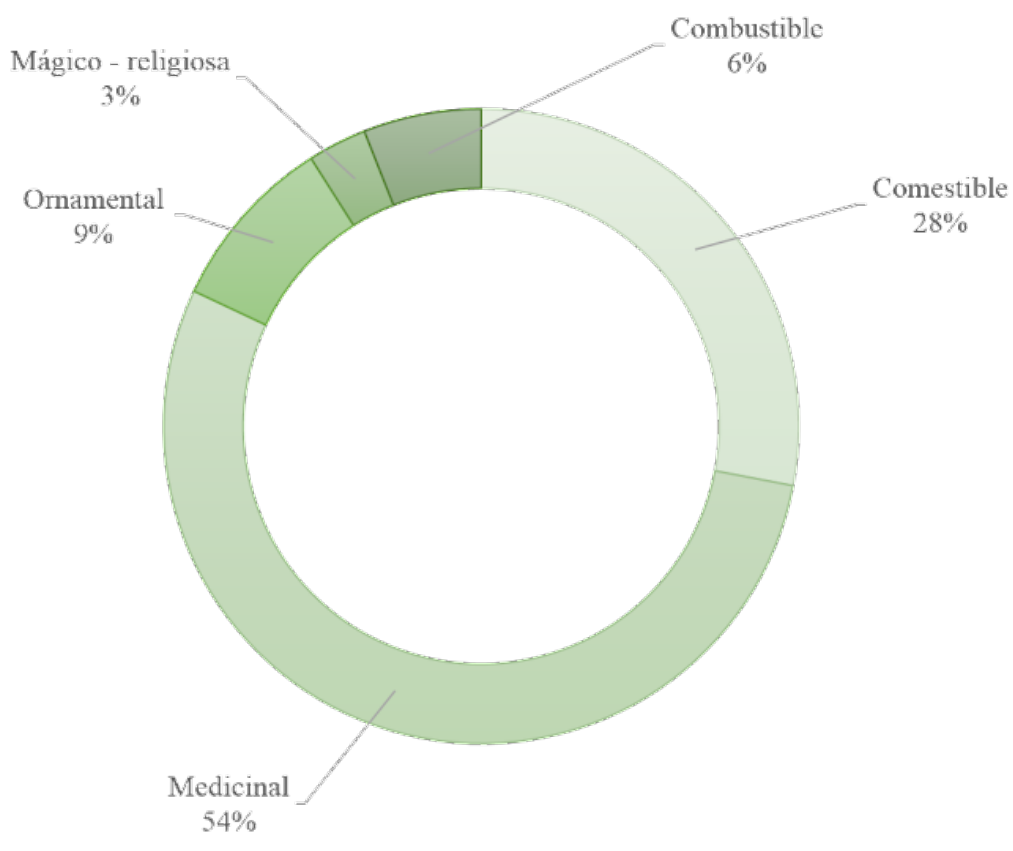




\section{Tabla 1}

Especies representativas y sus categorías de uso

\begin{tabular}{|c|c|c|c|c|c|c|}
\hline \multirow[b]{2}{*}{ Nombre común } & \multirow[b]{2}{*}{ Nombre científico } & \multicolumn{5}{|c|}{ Categorías de uso * } \\
\hline & & Co & $\mathrm{Me}$ & Or & Má-re & $\mathrm{Ma}$ \\
\hline Árnica & Arnica sp & & $\mathrm{X}$ & & & \\
\hline Alache & Anoda crisanta & $\mathrm{X}$ & & & & \\
\hline Albahaca & Ocimum basilicum & $\mathrm{x}$ & $\mathrm{X}$ & & & \\
\hline Azumiate & Senecio $s p$ & & $\mathrm{X}$ & & & \\
\hline Bugambilia & Bougainvillea spectabilis & & $\mathrm{X}$ & & & \\
\hline Calabaza & Cucurbita pepo & $\mathrm{x}$ & & & & \\
\hline Casahuate & Ipomoea murucoides & & $\mathrm{X}$ & & & $\mathrm{X}$ \\
\hline Cempasúchil & Tagetes erecta & & $\mathrm{X}$ & $\mathrm{X}$ & $\mathrm{X}$ & \\
\hline Chicali & Argemone mexicana $L$. & & $\mathrm{X}$ & & & \\
\hline Ciruela & Prunus domestica & $\mathrm{x}$ & & & & \\
\hline Encino & Quercus sp & & & & & $\mathrm{X}$ \\
\hline Frijol & Phaseolus vulgaris & $\mathrm{x}$ & & & & \\
\hline Granada & Punica granatum & $\mathrm{x}$ & $\mathrm{X}$ & & & \\
\hline Guajilote & Parmentiera edulis & $\mathrm{x}$ & $\mathrm{X}$ & & & \\
\hline Guayaba & Psidium guajava & $\mathrm{x}$ & $\mathrm{X}$ & & & \\
\hline Hierbabuena & Mentha piperita & $\mathrm{x}$ & $\mathrm{X}$ & & & \\
\hline Higuerilla & Ricinus communis & & $\mathrm{X}$ & $\mathrm{x}$ & & \\
\hline Huele de noche & Cestrum nocturnum & & $\mathrm{X}$ & & & \\
\hline Hierba santa & Piper auritum & $\mathrm{x}$ & & & & \\
\hline Laurel & Nerium oleander & $\mathrm{x}$ & $\mathrm{X}$ & & $\mathrm{X}$ & \\
\hline Limón & Citrus limon & $\mathrm{x}$ & $\mathrm{X}$ & & & \\
\hline Maíz & Zea mays & $\mathrm{x}$ & & & $\mathrm{X}$ & $\mathrm{X}$ \\
\hline Manrubio & Marrubium vulgare & & $\mathrm{X}$ & & & \\
\hline Manzanilla & Matricaria recutita & & $\mathrm{X}$ & & & \\
\hline Mezquite & Prosopis laevigata & & $\mathrm{X}$ & & & \\
\hline Noche buena & Euphorbia pulcherrima & & $\mathrm{X}$ & $\mathrm{x}$ & & \\
\hline Orégano & Lippia berlandieri & $\mathrm{x}$ & $\mathrm{X}$ & & & \\
\hline Pirul & Schinus molle & & $\mathrm{X}$ & & $\mathrm{X}$ & \\
\hline Romero & Rosmarinus officinalis & $\mathrm{x}$ & $\mathrm{X}$ & & & \\
\hline Ruda & Ruta chalepensis & & $\mathrm{X}$ & & & \\
\hline Sábila & Aloe barbadensis & & $\mathrm{X}$ & & & \\
\hline Santa María & Chysanthemum parthenium & & $\mathrm{X}$ & & & \\
\hline Sauco & Sambucus mexicana & & & & & \\
\hline Tronadora & Tecoma stans & & $\mathrm{X}$ & & & \\
\hline Yoyote & Thevetia thevetioides & & $\mathrm{X}$ & & & \\
\hline Zacatechichi & Calea ternifolia & & $\mathrm{X}$ & & & \\
\hline
\end{tabular}

Nota. * Categorías de uso: Comestible (Co), Medicinal (Me), Ornamental (Or), Mágico religiosas (Má-re) y Maderable (Ma). 
El conocimiento y el uso de plantas medicinales puede encontrarse desde lo general en cualquier tipo de hogar con especies más comunes como la hierbabuena o el romero (especies que también se encuentran como comestibles y mágico-religiosas), hasta lo especializado, es decir con especialistas de la medicina tradicional como hueseros, parteras o curanderos con especies como el zacatechichi o el azumiate (especies exclusivamente medicinales). De esta manera, la composición vegetal del huerto familiar puede variar según el oficio del dueño o de la familia que lo posee. Siguiendo este ejemplo, la diversidad de los huertos de traspatio en los hogares de dichos especialistas de la medicina tradicional pueden presentar un mayor número de especies de plantas con fines medicinales, mientras que el resto de la población tendrá una mayor cantidad de especies de las otras cuatro categorías dentro de sus huertos familiares de traspatio.

Los huertos representan una estrategia importante de la seguridad alimentaria por la disposición inmediata de los recursos. Sobre la alimentación Cano Contreras (2015, p. 79) indica que "es uno de los factores que condicionan el bienestar físico, la salud y la calidad de vida de las poblaciones". Por su parte Cahuich (2012) citado por Cano Contreras (2015) agrega que:

Cuando se analiza la alimentación de un pueblo o cultura, la necesidad de alimentos no debe ser entendida únicamente desde una dimensión biológica, sino enmarcada en una estrategia campesina compleja de uso múltiple de los recursos naturales, dada en el marco de la cosmovisión local, que permite a los grupos sociales identificarse, construir lazos de reciprocidad, compartir y generar conocimientos (pp. 78-79).

De esta cuenta, dentro de la comunidad de Xalpatláhuac pueden observarse dinámicas de intercambio a nivel familiar o vecinal principalmente con especies frutales, como el limón o la granada, que en algunos casos pueden significar un exceso de alimentos para una sola familia, o mediante festividades colectivas (como bodas, clausuras escolares o fiestas patronales) donde las diferentes familias participantes aportan distintos productos como chiles, aguacates, cebollas, tomates y jitomates para la preparación de los alimentos, y que al encontrarse dentro de sus huertos familiares representan un menor gasto económico para la contribución de cada familia.

Desde la perspectiva de los habitantes de la comunidad de Xalpatláhuac tener un huerto dentro de sus hogares representa un apoyo muy importante para complementar necesidades básicas como la alimentación o dentro del primer nivel de atención ante algún padecimiento en la salud como algún resfriado o una quemadura leve, lo cual en otras circunstancias representaría un gasto económico que muchas familias no podrían solventar. Además, muchas personas, sobre todo la gente mayor, considera importante conocer el origen de los alimentos que se consumen, de esta manera pueden estar seguros que los recursos que utilizan directo del huerto no contienen restos de pesticidas o fertilizantes químicos que puedan generar con el tiempo daños considerables a la salud. Otras personas también hacen mención de que al tener un huerto dentro de sus hogares les permite poder ir seleccionando las mejores variedades de plantas por su tamaño, su color o su sabor, mejorando con el tiempo algunas plantas y teniendo cosechas que se adecuan a los gustos particulares de las familias.

\section{Estructura de los huertos}

El huerto familiar para los habitantes de Xalpatlahuac es el espacio ubicado preferentemente en el traspatio de la casa habitación, allí siembran sus plantas o las mantienen en macetas u otro tipo de contenedores como ollas o huacales (cajas de madera utilizados para transportar frutas), algunos huertos familiares cumplen la doble función con parcela y la gran mayoría con labores agrícolas como es el secado de semillas, desgrane, manejo de rastrojo e incluso como puntos de comercialización.

Haciendo una diferenciación general, es muy frecuente encontrar especies grandes como árboles frutales o arbustos medianos sembrados directamente sobre el suelo de tierra, entre los primeros podemos encontrar especies como la naranja (Citrus sinensis), la granada (Punica granatum), el limón (Citrus limon), el guajilote (Parmentiera edulis) o el huamuchil (Pithecellobium dulce), mientras que entre los principales arbustos que se encuentran está el estafiate (Artemisia ludoviciana), el azumiate (Baccharis salicifolia), la bugambilia (Bougainvillea $s p$ ) y la nochebuena (Euphorbia pulcherrima). Por otro lado, las plantas que se encuentran en mayor medida en ollas, macetas o huacales son especies pequeñas o rastreras como la hierbabuena (Mentha spicata), la menta (Mentha piperita), el jitomate (Physalis philadelphica) el tomate (Solanum lycopersicum), o diferentes variedades de 
chile (Capsicum sp). Sin embargo, existen algunas especies que pese a ser pequeñas se encuentran directamente sobre el suelo, esto debido a su carácter "silvestre" que por lo general no permite su permanencia en algún tipo de contenedor o recipiente, entre estas encontramos el manrubio (Marrubium vulgare), el zacatechichi (Calea ternifolia) y el epazote (Dysphania ambrosioides).

Respecto a la disposición de los huertos, cabe resaltar que la arquitectura de las casas es diversa y se encuentra construido de acuerdo a la topografía accidentada de la comunidad, el orden generalmente se encuentra de la siguiente manera: las especies que se encuentran en macetas, huacales y ollas se encuentran en la periferia, a un lado de las paredes o bardas (Figura 4) y en algunos casos en las ventanas, los árboles frutales se encuentran distribuidos estratégicamente a lo largo del patio para generar sombra, los más grandes en la periferia y los medianos al centro, mientras que las plantas medianas y pequeñas que se encuentran directamente sobre la tierra se ubican bajo los árboles medianos.

La migración también es un factor determinante en la infraestructura de los hogares y sus componentes, ya que desde los años setenta del siglo pasado aumentó significativamente, llevando a las comunidades a un contexto rural en vías de urbanización (Figura 5). De acuerdo con lo comentado en la comunidad, el cambio estructural de las casas se comenzó a presentar después del año 2000, con el paso del tiempo las construcciones han variado y aumentado; en la actualidad Xalpatláhuac es la única comunidad a la que se le atribuye el cambio de categoría de rural a urbano (Secretaría de Desarrollo Social [SEDESOL], 2013).

Lo anterior se expresa en los cambios estructurales en los huertos familiares, por un lado, hay huertos donde el $70 \%$ de las plantas se encuentran en el suelo y el resto en contenedores (cubetas, macetas, huacales, trastes de barro, aluminio o peltre) (Figura 6), estos se encuentran en gran mayoría en las casas con menos modificaciones estructurales o de remodelación urbana. Caso contrario con las casas urbanizadas en ellas el $80 \%$ a $90 \%$ de las plantas se encuentran en contenedores y sólo unos cuantos ejemplares (principalmente árboles o arbustos) en el suelo, debido a que la mayoría de las casas colocan pisos de cemento, reduciendo el espacio para sembrar plantas. Los cambios estructurales también se reflejan en el número de especies presentes, ya que las casas con más espacio de jardín o con menos remodelaciones urbanas presentan aproximadamente $30 \%$ más plantas que las casas urbanizadas.

\section{Figura 5}

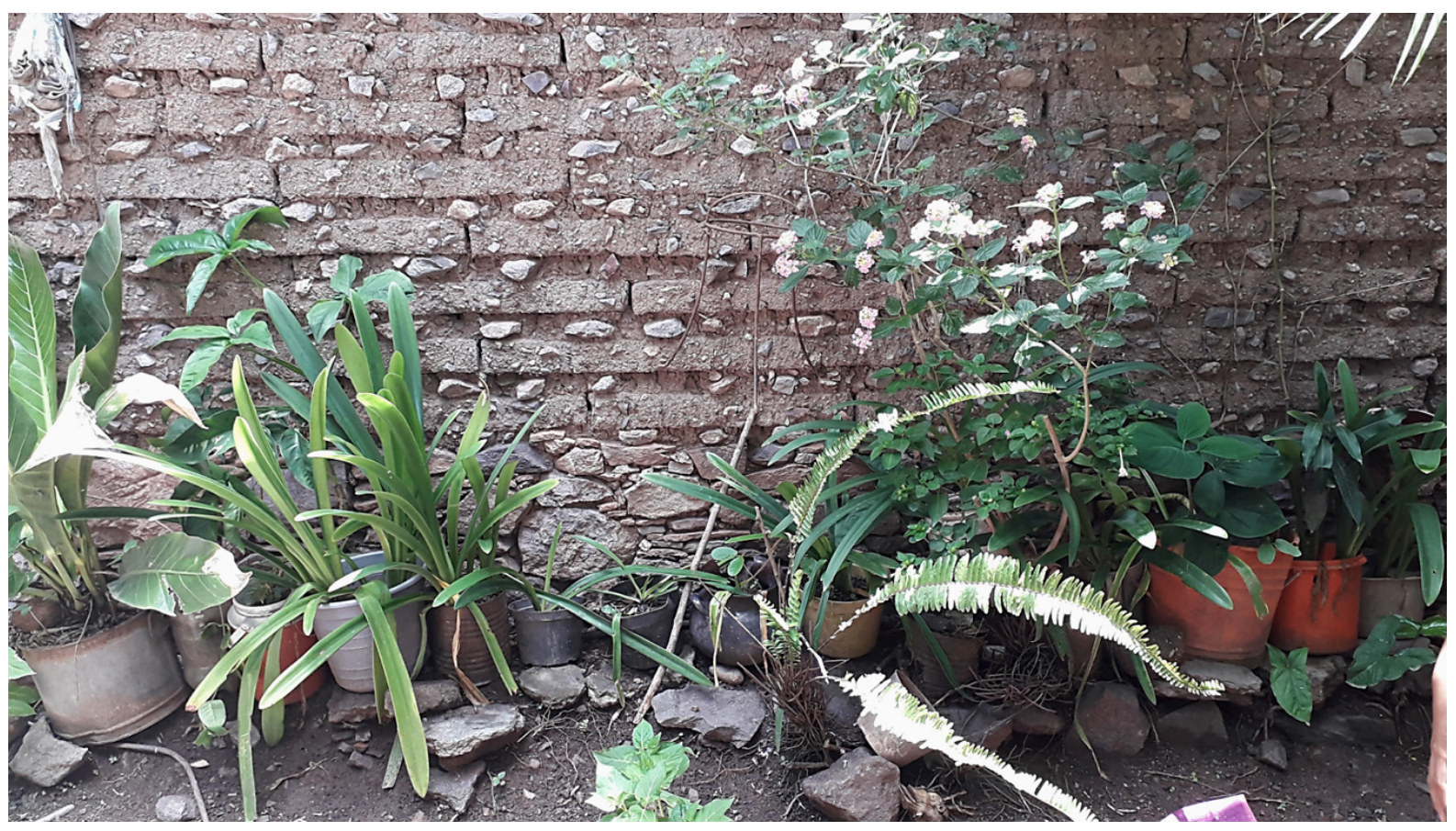




\section{Figura 6}

Cambio del modelo de casas tradicionales al modelo urbano. Al frente pueden observarse restos de las paredes de adobe de la casa tradicional que se derrumbó para construir el nuevo modelo

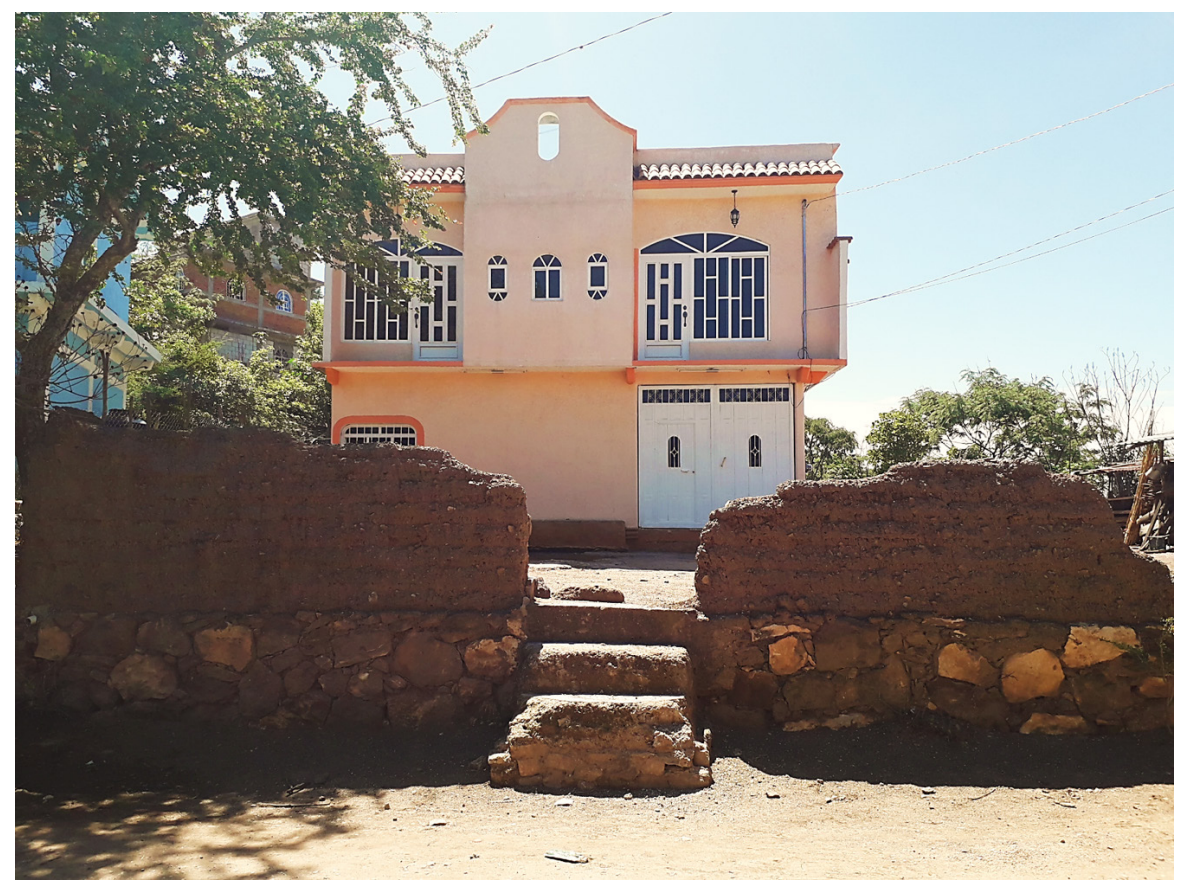

\section{Figura 7}

Uso de macetas, ollas y otros recipientes para la siembra de plantas
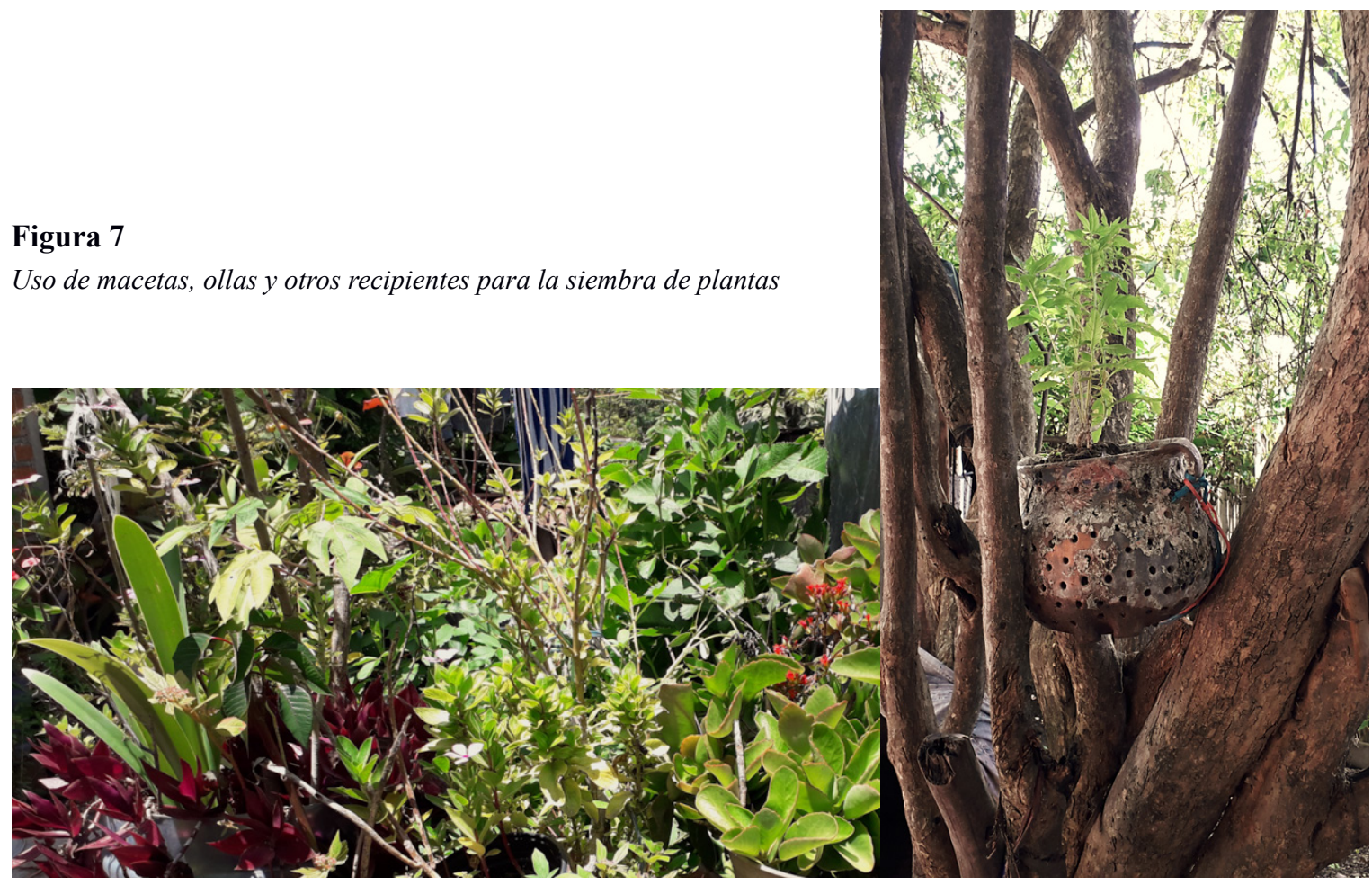


\section{Importancia económica}

La principal práctica económica derivada del huerto familiar es el autoconsumo, en el caso particular de que existan excedentes se realiza el intercambio y la venta del recurso en bruto o transformado. El intercambio sólo se ejecuta con familiares o vecinos, y dentro de estos intercambios podemos encontrar especies como distintos tipos de chiles (Capsicum $s p$ ), chayote (Sechium edule), o frutos como la naranja ( $\mathrm{C} i$ trus sinensis) o la guayaba (Psidium guajava), la venta se realiza de forma ambulante por la zona comercial, colocando una mesa afuera del hogar o en las escuelas en forma de bolis (congelada de fruta) o fruta picada, en este caso podemos encontrar especies como el huamuchil (Pithecellobium dulce), el nanche (Byrsonima crassifolia), el limón (Citrus limon) o el mango (Mangifera sp).

\section{Prácticas socioculturales}

Los huertos familiares son resultado de la herencia social en un lugar, Xalpatláhuac no es la excepción. Esto ha sido posible después de un alto costo social, al hacer una gran inversión de trabajo año tras año en la selección de las variantes con mutaciones favorables a la cosecha, el consumo y el manejo, y al cotejo del comportamiento productivo de las variantes selectas. (Zizumbo-Villarreal \& Colunga-GarcíaMarín, 2017). Lo anterior se ve reflejado en la permanencia de las plantas útiles nativas silvestres de la región y en las múltiples referencias de la transmisión de conocimiento horizontal, ejemplo de lo anterior son las expresiones de "así nos enseñaron" o "así lo comían los abuelitos" con plantas como el guajilote (Parmentiera aculeata), los alaches (Anoda cristata) y la ciruela (Prunus domestica). Este tipo de prácticas son enseñadas a los niños desde pequeños cuando acuden al campo, a quienes se les enseña a diferenciar entre las especies comestibles y aquellas que no lo son, así como las distintas etapas de maduración y recolección de las plantas útiles, y en algunos casos también se les enseñan diferentes tipos de reproducción asexual de las plantas como acodos y esquejes; sin embargo, esto último es cada vez menos común.

\section{Participación de las mujeres}

La existencia, gestión y mantenimiento del huerto es producto de las mujeres, lo anterior no es "coin- cidencia", ya que ellas son las encargadas del abastecimiento alimentario en el hogar. Esto también es reportado por (Cruz Yánez, 2016) quien puntualiza la participación de la mujer desde el establecimiento del huerto hasta la cosecha de los productos. En la comunidad se vive la división del trabajo por rol de género, es decir, el trabajo es diferenciado a partir de características genéricas previamente establecidas. Es un fenómeno evidente, dado que la mayoría de las entrevistadas mencionaron que los maridos que saben de plantas responden en el ámbito de la milpa o el monte, mientras que el hogar y sus huertos eran tarea de ellas.

A pesar de que se argumenta que hay una estructura de las labores relacionadas con el huerto con base en el género (predominando el género femenino), se sugiere que el conocimiento no es específico de ellas y que hay una fuerte transmisión del conocimiento a los niños y las niñas, quienes apoyan sobre todo en el riego y cosecha de frutos y hojas, sin embargo por la división del trabajo dentro y fuera del hogar, el mantenimiento del huerto queda en manos de las mujeres, aunque de ser necesario los hombres también pueden intervenir, sobre todo en la siembra de plantas nuevas, o poda de árboles.

Respecto a los alimentos que se preparan con productos del huerto destacan la preparación de salsas con diferentes tipos de chile (Capsicum sp), cebolla (Allium cepa) y ajo (Allium sativum), las cuales se preparan casi todos los días, o guisos como los alaches (Anoda cristata) o quiltoniles (Amaranthus sp), los cuales se hierven o se fríen y se acompañan con tortillas, así como la preparación de bebidas frutales como el agua de mango (Mangifera sp), naranja (Citrus sinensis), limón (Citrus limon) o guayaba (Psidium guajava), las cuales acompañan las comidas. Por las noches suelen prepararse bebidas calientes principalmente con zacate limón (Cymbopogon citratus), manzanilla (Matricaria recutita), canela (Cinnamomum verum), hojas de naranjo (Citrus sinensis) o limón (Citrus limon). También son utilizadas diferentes especies a modo de especia, para dar sabor a distintos alimentos como caldos, sopas y carnes, como en el caso del comino (Cuminum cyminum), la pimienta (Piper nigrum) o el epazote (Dysphania ambrosioides).

\section{Comentarios finales}

Dado que los sistemas de manejo se encuentran en constante modificación en función de las necesidades humanas, estudiar los huertos familiares tradicionales 
nos permite conocer y entender el conocimiento asociado a las prácticas de manejo tradicionales que preservan las especies nativas y domesticadas. Aún hace falta conocer a profundidad los métodos de manejo en la comunidad en todos los sistemas agroforestales tradicionales presentes.

La diversidad de plantas permite a las familias atender afecciones a su salud y alimenticias, además que representa un recurso de fácil acceso y único recurso ante la falta de recursos económicos. Los resultados resaltan la importancia de la flora medicinal por encima de la flora comestible en la comunidad, esto nos indica que el conocimiento medicinal tradicional continúa practicándose a través de las generaciones.

Con este trabajo se reafirma el nivel organizativo de las mujeres en el aprovechamiento, intercambio y cuidado de los recursos respondiendo a la necesidad de complementar con productos obtenidos del huerto familiar y no únicamente con productos obtenidos del campo.

\section{Agradecimientos}

A las personas de Xalpatláhuac que compartieron su tiempo, conocimiento y espacios con nosotros, por siempre mostrar calidez y apoyo en la realización de este trabajo.

A la Brigada Multidisciplinaria de Apoyo a las Comunidades de México e Infrarural por permitirnos conocer y trabajar en la comunidad.

A todos aquellos que participaron en el proyecto de agroecología, de la BMACM, por su colaboración en la recolección de datos que nos entregó la base para este estudio.

\section{Referencias}

Cadena I., Rendón-Medel, R., Aguilar-Ávila, J., Eileen Salinas-Cruz, J., Francisca del Rosario de la Cruz-Morales, F., \& Dora Ma. Sangerman-Jarquín, D. M. (2017). Métodos cuantitativos, métodos cualitativos o su combinación en la investigación: Un acercamiento en las ciencias sociales. Revista Mexicana de Ciencias Agrícolas, 8(7), 1603-1617. https://doi.org/10.29312/remexca. v8i7.515

Cano Contreras, E. J. (2015). Huertos familiares: Un camino hacia la soberanía alimentaria. Revista Pueblos y Fronteras, 10(20), 70-91. https://doi. org/10.22201/cimsur.18704115e.2015.20.33
Casas, A., \& Parra, F. (2016). La domesticación como proceso evolutivo. En A. Casas, J. Torres-Guevara \& F. Parra (Eds.), Domesticación en el continente americano (Vol. 1, pp. 133-158). Universidad Nacional Autónoma de México, Universidad Nacional Agraria de la Molina.

Chávez, C., Arriaga, C. \& González, C. (2002). Uso y manejo de la flora del solar en dos comunidades mazahuas. En G. Rivera (Coord.), Investigación para el desarrollo rural: Nuevas experiencias a $X V$ años del CICA (pp. 25-55). Universidad Autónoma del Estado de México.

Cruz Yáñez, L. A. (2016). El papel de las mujeres en los huertos familiares. Alternativas en psicología, (36), 46-60.

Gispert, M., Gómez, A. \& Núñez A. (1993). Concepto $\mathrm{y}$ manejo tradicional de los huertos familiares en dos bosques tropicales mexicanos. En E. Leff, \& J. Carabias (Coords.), Cultura y manejo sustentable de los recursos naturales (Vol. 2, pp. 576-623). Porrúa, Centro de Investigaciones Interdisciplinarias en Humanidades, Universidad Nacional Autónoma de México

Hamui-Sutton, A., \& Varela-Ruiz, M. (2013). La técnica de grupos focales. Revista Investigación en Educación Médica, 2(5), 55-60. https://doi. org/10.1016/S2007-5057(13)72683-8

Instituto de Ecología. (2021). El patrimonio biocultural de México; un tesoro de los pueblos indígenas. https://www.inecol.mx/inecol/index.php/ es/2017-06-26-16-35-48/17-ciencia-hoy/784-elpatrimonio-biocultural-de-mexico-un-tesoro-d los-pueblos-indigenas

Instituto Nacional de Estadística y Geografía. (2015). Panorama sociodemográfico de Guerrero, Municipio de Xalpatláhuac. Recuperado de http:// internet.contenidos.inegi.org.mx/contenidos/ productos//prod_serv/contenidos/espanol/bvinegi/productos/nueva_estruc/inter_censal/panora$\mathrm{ma} / 702825082208 . \mathrm{pdf}$

Instituto Nacional para el Federalismo y el Desarrollo Municipal. (2020). Enciclopedia de los Municipios y Delegaciones de México. Estado de Guerrero. Recuperada de http://www.inafed.gob. $\mathrm{mx} /$ work/enciclopedia/EMM12guerrero/municipios/12069a.html 
López Pérez, F. (2017). Contribución de los huertos familiares biointensivos al desarrollo sostenible de las familias rurales y periurbanas [Tesis de maestría]. Recuperada de https://www. researchgate.net/profile/Rita-Rindermann/publication/319874109_Huertos_familiares_en Mexocp/links/59bf001e458515e9cfd22e7a/Huertos-familiares-en-Mexocp.pdf

Mariaca Méndez, R., Álvarez Ávila, M. C., Arias Reyes, L. M., Cahuich-Campos, D., González Jácome, A., Vásquez Dávila, M. A., \& van der Wall, H. (2010). Avances en el estudio de los huertos familiares del sur de México. En A. Moreno Fuentes, M. T. Pulido Silva, R. Mariaca Méndez, R. Valadez Azúa, P. Mejía Correa, \& T. V. Gutiérrez Santillán (Eds.), Sistemas biocognitivos tradicionales, paradigmas en la conservación biológica y el fortalecimiento cultural (pp. 108-116). Asociación Etnobiológica Méxicana, Global Diversity Foundation, Universidad Autónoma del Estado de Hidalgo, El Colegio de la Frontera Sur, Sociedad Latinoamericana de Etnobiología.

Moreno-Calles, A. I., Galicia-Luna, V. J., Casas, A., Toledo, V.M., Vallejo-Ramos, M., Santos-Fita, D., \& Camou-Guerrero, A. (2014). La etnoagroforestería: El estudio de los sistemas agroforestales tradicionales de México. Etnobiología, 12(3), 2-16.

Moreno Calles, A. I., Casas, A., Toledo, V. M., \& Vallejo Ramos, M. (2016). Introducción. Etnoagroforestería en México, los proyectos y la idea del libro. En A. I. Moreno Calles, A. Casas, V.M. Toledo, M. Vallejo (Comps.). Etnoagroforestería en México (pp. 11-23). Universidad Nacional Autónoma de México.

Ramírez Bacca, R. (2010). Introducción teórica y práctica a la investigación histórica. Guía para historia en ciencias sociales. Universidad Nacional de Colombia.

Rzedowski, R. (1978). Vegetación de México. Limusa.

Secretaria de Desarrollo Social. (2013). Catálogo de localidades. Recuperado de http://www.microrregiones.gob.mx/catloc/LocdeMun.aspx?tipo=clave $\&$ campo $=$ loc $\&$ ent $=12 \&$ mun $=069 \&$ fbclid $=I-$ wAR2zL3cEMvdCMbKh6m1LJgc4T9mUKHrSv-iHQaSFwstXM792Zo76PJS_CWc
Sociedad Latinoamericana de Etnobiología. (2016). Código de Ética para la investigación, la investigación-acción y la colaboración etnocientífica en América Latina. Etnobiología. 14(4), 3-30.

Vallejo-Ramos, M., Moreno-Calles, A., \& Casas, A. (2016). TEK and biodiversity management in agroforestry systems of different socio-ecological contexs of the Tehuacán Valley. Journal of Ethnobiology and Ethnomedicine, 12(31), 1-15. https:// doi.org/10.1186/s13002-016-0102-2

Zizumbo-Villarreal, D., \& Colunga-GarcíaMarín, P.(2017). El sistema agroalimentario mesoamericáno precolombino: orígen, diversificación y difusión de sus recursos fitogenéticos. En A. Casas, J. Torres-Guevara \& F. Parra (Eds.). Domesticación en el continente americano, (Vol. 2, pp. 375389). Universidad Nacional Autónoma de México, Universidad Nacional Agraria La Molina. 